\title{
On the $\ell_{1}$-Norm Invariant Convex $k$-Sparse Decomposition of Signals
}

\author{
Guangwu Xu • Zhiqiang Xu
}

Received: 17 October 2013 / Revised: 11 November 2013 / Accepted: 19 November 2013 /

Published online: 3 December 2013

(C) Operations Research Society of China, Periodicals Agency of Shanghai University, and

Springer-Verlag Berlin Heidelberg 2013

\begin{abstract}
Inspired by an interesting idea of Cai and Zhang, we formulate and prove the convex $k$-sparse decomposition of vectors that is invariant with respect to the $\ell_{1}$ norm. This result fits well in discussing compressed sensing problems under the Restricted Isometry property, but we believe it also has independent interest. As an application, a simple derivation of the RIP recovery condition $\delta_{k}+\theta_{k, k}<1$ is presented.
\end{abstract}

Keywords Convex $k$-sparse decomposition $\cdot \ell_{1}$ minimization $\cdot$ Restricted isometry property $\cdot$ Sparse recovery

\section{Introduction}

The Restricted Isometry Property (RIP) of Candès and Tao [7] is one of the most commonly used frameworks for sparse recovery via $\ell_{1}$ minimization. For an $n \times p$ matrix $\Phi \in \mathbb{R}^{n \times p}$ and an integer $k, 1 \leqslant k \leqslant p$, the $k$-restricted isometry constant $\delta_{k}$ is the smallest constant such that

$$
\sqrt{1-\delta_{k}}\|c\|_{2} \leqslant\|\Phi c\|_{2} \leqslant \sqrt{1+\delta_{k}}\|c\|_{2}
$$

This research was partially supported by the National 973 Project of China (No. 2013CB834205). Zhiqiang Xu was supported by National Natural Science foundation of China (Nos. 11171336, 11331012, 11021101) and National Basic Research Program of China (973 Program No. 2010CB832702).

G. Xu

Department of EE \& CS, University of Wisconsin-Milwaukee, Milwaukee, WI 53211, USA

e-mail: gxu4uwm@uwm.edu

Z. $\mathrm{Xu}(\bowtie)$

Inst. Comp. Math., Academy of Mathematics and Systems Science, Chinese Academy of Sciences,

Beijing, China

e-mail: xuzq@1sec.cc.ac.cn 
for every $k$-sparse vector $c$ (namely, $c$ has at most $k$ nonzero components). If $k+k^{\prime} \leqslant p$, the $k, k^{\prime}$-restricted orthogonality constant $\theta_{k, k^{\prime}}$, is the smallest number that satisfies

$$
\left|\left\langle\Phi c, \Phi c^{\prime}\right\rangle\right| \leqslant \theta_{k, k^{\prime}}\|c\|_{2}\left\|c^{\prime}\right\|_{2},
$$

for all $k$-sparse vector $c$ and $k^{\prime}$-sparse vector $c^{\prime}$ with disjoint supports.

It has been shown that $\ell_{1}$ minimization can recover a sparse signal with a small or zero error under various conditions on $\delta_{k}$ and $\theta_{k, k^{\prime}}$, such as the condition $\delta_{k}+\theta_{k, k}+$ $\theta_{k, 2 k}<1$ in Candès and Tao [7], and the condition $\delta_{k}<0.307$ of Cai, Wang and $\mathrm{Xu}$ [6]. Recently, Cai and Zhang [2] established a sharp condition on $\delta_{k}$ for $k$-sparse recovery:

$$
\delta_{k}<\frac{1}{3} .
$$

In the same paper, they also proved that $\delta_{2 k}<\frac{1}{2}$ is sufficient for $k$-sparse signal reconstruction. Cai and Zhang developed a marvelous technique in the proof of their results.

Inspired by the division lemma of Cai and Zhang [2], we formulate and prove the $\ell_{1}$-norm invariant convex $k$-sparse decomposition of vectors in this note. This result (Theorem 2.1) asserts that every vector is a convex combination of $k$-sparse vectors with invariant $\ell_{1}$ norm. Such decomposition fits well in treating compressed sensing problems under RIP, as a tighter conversion between $\ell_{1}$-norm and $\ell_{2}$-norm is desired. We shall demonstrate this by showing how to use the decomposition to derive the sparse recovery condition $\delta_{k}+\theta_{k, k}<1$ of Cai and Zhang [3] in a simple manner. However, we believe that this result is of independent interest for other applications.

After the early appearance of this note (arXiv:1305.6021, May 2013), we learned that Cai and Zhang [4] also established a similar decomposition and using it derived some good RIP conditions (e.g., $\delta_{2 k}<\frac{1}{\sqrt{2}}$ ). Using the $\ell_{1}$-norm invariant convex $k$-sparse decomposition, under the tight frame sparsification, Baker [1] obtained the condition $\delta_{2 k}<\frac{1}{\sqrt{2}}$ for the Dictionary-Restricted Isometry Property.

The paper is organized as follows. Section 2 presents the $\ell_{1}$-norm invariant $k$ sparse convex decomposition. As a consequence of this decomposition, we prove a useful result for comparing $\ell_{p}$-norms. In Sect. 3 , the $k$-sparse convex decomposition is used to give a simple derivation of the sparse recovery condition $\delta_{k}+\theta_{k, k}<1$ of Cai and Zhang.

\section{Convex $k$-Sparse Decomposition}

In this section, we prove that every vector is a convex combination of $k$-sparse vectors with invariant $\ell_{1}$-norm. The formulation is inspired by the celebrated ideas from Cai and Zhang [2]. We also show that the $\ell_{\infty}$-norm of the summand vectors is well behaved. More specifically, we have

Theorem 2.1 For positive integers $k \leqslant n$, and positive constant $C$, let $\mathbf{v} \in \mathbb{R}^{n}$ be a vector with

$$
\|\mathbf{v}\|_{1} \leqslant C \quad \text { and } \quad\|\mathbf{v}\|_{\infty} \leqslant \frac{C}{k}
$$


Then there are $k$-sparse vectors $\mathbf{w}_{1}, \cdots, \mathbf{w}_{M}$ with

$$
\left\|\mathbf{w}_{t}\right\|_{1}=\|\mathbf{v}\|_{1} \quad \text { and } \quad\left\|\mathbf{w}_{t}\right\|_{\infty} \leqslant \frac{C}{k} \quad \text { for } t=1, \cdots, M,
$$

such that

$$
\mathbf{v}=\sum_{t=1}^{M} x_{t} \mathbf{w}_{t}
$$

for some nonnegative real numbers $x_{1}, \cdots, x_{M}$ with $\sum_{t=1}^{M} x_{t}=1$.

Proof If $k=n$, or $\mathbf{v}$ is already $k$-sparse, then there is nothing to do. Assume now $n>k$. Without loss of generality, we may consider the case that all components of $\mathbf{v}$ are positive $^{1}$ (the general case can be argued easily, as (2.2) still holds by multiplying -1 to the $i$ th components of both sides):

$$
\mathbf{v}(1) \geqslant \mathbf{v}(2) \geqslant \cdots \geqslant \mathbf{v}(n)>0 .
$$

For each $j=1, \cdots, k$, let $\eta_{j}:=\frac{C}{k}-\mathbf{v}(j)$. Since $\sum_{j=1}^{k} \eta_{j}=C-\mathbf{v}(1)-\cdots-$ $\mathbf{v}(k) \geqslant \mathbf{v}(k+1)+\cdots+\mathbf{v}(n)$, so we have $\eta_{j} \geqslant 0$ and $\sum_{j=1}^{k} \eta_{j}>0$. Let

$$
\lambda_{i}:=\frac{\eta_{i}}{\sum_{j=1}^{k} \eta_{j}}, \quad i=1,2, \cdots, k
$$

Then $\sum_{i=1}^{k} \lambda_{i}=1$.

We shall construct $k+1$ vectors $\mathbf{g}_{0}, \cdots, \mathbf{g}_{k}$ : each has $n-1$ nonzero components and satisfies

$$
\left\|\mathbf{g}_{t}\right\|_{1}=\|\mathbf{v}\|_{1} \quad \text { and } \quad\left\|\mathbf{g}_{t}\right\|_{\infty} \leqslant \frac{C}{k} \quad \text { for } t=0,1, \cdots, k .
$$

Furthermore, $\mathbf{v}$ is a convex combination of $\mathbf{g}_{0}, \cdots, \mathbf{g}_{k}$. In the following construction, we will use $\mathbf{v}_{\{j\}}$ to denote the vector whose $j$ th component is $\mathbf{v}(j)$ and other components are zero, and use $\left\{\mathbf{e}_{1}, \cdots, \mathbf{e}_{n}\right\}$ to denote the canonical basis. The $k+1$ vectors $\mathbf{g}_{0}, \cdots, \mathbf{g}_{k}$ are

$$
\begin{aligned}
& \mathbf{g}_{0}=\sum_{j=1}^{k}\left(\mathbf{v}(j)+\lambda_{j} \mathbf{v}(n)\right) \mathbf{e}_{j}+\mathbf{v}_{\{k+1\}}+\cdots+\mathbf{v}_{\{n-1\}}, \\
& \mathbf{g}_{t}=\sum_{\substack{j=1 \\
j \neq t}}^{k}\left(\mathbf{v}(j)+\lambda_{j} \mathbf{v}(n)\right) \mathbf{e}_{j}+\left(\mathbf{v}(t)+\lambda_{t} \mathbf{v}(n)\right) \mathbf{e}_{n}+\mathbf{v}_{\{k+1\}}+\cdots+\mathbf{v}_{\{n-1\}}, 1 \leqslant t \leqslant k .
\end{aligned}
$$

Let

$$
y_{1}:=\frac{\lambda_{1} \mathbf{v}(n)}{\mathbf{v}(1)+\lambda_{1} \mathbf{v}(n)}, \quad y_{2}:=\frac{\lambda_{2} \mathbf{v}(n)}{\mathbf{v}(2)+\lambda_{2} \mathbf{v}(n)}, \quad \cdots, \quad y_{k}:=\frac{\lambda_{k} \mathbf{v}(n)}{\mathbf{v}(k)+\lambda_{k} \mathbf{v}(n)} .
$$

\footnotetext{
${ }^{1}$ In this note, we will treat a vector of $\mathbb{R}^{n}$ as a function from $\{1, \cdots, n\}$ to $\mathbb{R}$.
} 
Then we see that $y_{i}<\lambda_{i}$. So, by setting $y_{0}=1-y_{1}-\cdots-y_{k}$, we have $y_{0}>0$ and

$$
y_{0}+y_{1}+\cdots+y_{k}=1 \text {. }
$$

It is also straightforward to verify

$$
\mathbf{v}=y_{0} \mathbf{g}_{0}+y_{1} \mathbf{g}_{1}+\cdots+y_{k} \mathbf{g}_{k} .
$$

For example, the first component of $y_{0} \mathbf{g}_{0}+y_{1} \mathbf{g}_{1}+\cdots+y_{k} \mathbf{g}_{k}$ is

$$
\begin{aligned}
& y_{0}\left(\mathbf{v}(1)+\lambda_{1} \mathbf{v}(n)\right)+y_{2}\left(\mathbf{v}(1)+\lambda_{1} \mathbf{v}(n)\right)+\cdots+y_{k}\left(\mathbf{v}(1)+\lambda_{1} \mathbf{v}(n)\right) \\
& \quad=\left(\mathbf{v}(1)+\lambda_{1} \mathbf{v}(n)\right)\left(1-y_{1}\right)=\mathbf{v}(1) .
\end{aligned}
$$

The other requirements for $\mathbf{g}_{t}(t=0,1, \cdots, k)$ are:

1. $\left\|\mathbf{g}_{t}\right\|_{1}=\|\mathbf{v}\|_{1}$. This is certainly true.

2. $\left|\mathbf{g}_{t}(i)\right| \leqslant \frac{C}{k}$. To see this, we note that

$$
\mathbf{g}_{t}(i)= \begin{cases}0 & \text { if } i=t \text { or if } t=0, i=n \\ \mathbf{v}(i)+\lambda_{i} \mathbf{v}(n) & \text { if } 1 \leqslant i \leqslant k \text { and } i \neq t \\ \mathbf{v}(i) & \text { if } k<i<n \\ \mathbf{v}(t)+\lambda_{t} \mathbf{v}(n) & \text { if } t>0, i=n .\end{cases}
$$

For $1 \leqslant i \leqslant n$,

$$
\begin{aligned}
\frac{C}{k}-\left|\mathbf{g}_{t}(i)\right| & \geqslant \frac{C}{k}-\left(\mathbf{v}(i)+\lambda_{i} \mathbf{v}(n)\right)=\eta_{i}-\lambda_{i} \mathbf{v}(n) \\
& =\eta_{i}-\frac{\eta_{i} \mathbf{v}(n)}{\sum_{j=1}^{k} \eta_{j}} \geqslant \eta_{i}-\frac{\eta_{i} \mathbf{v}(n)}{\mathbf{v}(k+1)+\cdots+\mathbf{v}(n)} \geqslant 0 .
\end{aligned}
$$

If $n-1>k$, we repeat this process for each $\mathbf{g}_{t}$, and so on, until a $k$-sparse convex combination is reached.

Remark 2.2 The proof of Theorem 2.1 in fact presents a method to construct the vectors $\mathbf{w}_{t}, t=1, \cdots, M$, with $M=\left(\begin{array}{l}n \\ k\end{array}\right)$. Using this method, the time complexity to construct the $M$ vectors is $O\left(k^{n-k}\right)$. It will be interesting to design efficient algorithms to construct the vectors.

\section{RIP Conditions in Compressed Sensing}

In this section, we shall use the $k$-sparse convex decomposition to describe a short proof of the following results of Cai and Zhang [3]. Our proof follows an approach similar to that in $[5,6]$. We first consider the recovery of $k$-sparse signal:

Theorem 3.1 Let $\beta$ be a $k$-sparse signal and $y=\Phi \beta$ where $\Phi$ satisfies

$$
\delta_{k}+\theta_{k, k}<1 \text {. }
$$

Let

$$
\hat{\beta}=\underset{\gamma \in \mathbb{R}^{p}}{\arg \min }\left\{\|\gamma\|_{1} \text { subject to } y=\Phi \gamma\right\} .
$$

Then $\beta=\hat{\beta}$. 
Proof Let $h:=\hat{\beta}-\beta$. We need to show that $h=0$. Otherwise, we assume $|h(1)| \geqslant$ $|h(2)| \geqslant \cdots \geqslant|h(p)|>0$. Denote

$$
T:=\{1,2, \cdots, k\}, \quad S:=\{k+1, k+2, \cdots, p\},
$$

then as in [6], the minimality of $\hat{\beta}$ yields

$$
\left\|h_{S}\right\|_{1} \leqslant\left\|h_{T}\right\|_{1},
$$

where $h_{Q}=h \mathbb{I}_{Q}$ and $\mathbb{I}_{Q}$ denotes the indicator function of the set $Q$ (namely, $\mathbb{I}_{Q}(j)=$ 1 if $j \in Q$ and 0 if $j \notin Q$ ).

From the assumption, we also have $\left|h_{S}(j)\right| \leqslant \frac{\left\|h_{T}\right\|_{1}}{k}$ for all $j \in S$, i.e.

$$
\left\|h_{S}\right\|_{\infty} \leqslant \frac{\left\|h_{T}\right\|_{1}}{k} \text {. }
$$

Therefore, by Theorem 2.1, $h_{S}$ can be written as

$$
h_{S}=\sum_{j=1}^{M} x_{j} \mathbf{w}_{j} \quad \text { where } x_{j} \geqslant 0 \text { and } \sum_{j=1}^{q} x_{j}=1,
$$

where each $\mathbf{w}_{j}$ is $k$-sparse and supported on $S$, and $\left\|\mathbf{w}_{j}\right\|_{1}=\left\|h_{S}\right\|_{1},\left\|\mathbf{w}_{j}\right\|_{\infty} \leqslant \frac{\left\|h_{T}\right\|_{1}}{k}$. As $h_{T}$ and $\mathbf{w}_{j}$ have disjoint supports and $\left\|\mathbf{w}_{j}\right\|_{2} \leqslant \sqrt{k\left(\frac{\left\|h_{T}\right\|_{1}}{k}\right)^{2}} \leqslant\left\|h_{T}\right\|_{2}$, we get

$$
\begin{aligned}
\left(1-\delta_{k}\right)\left\|h_{T}\right\|_{2}^{2} & \leqslant\left\|\Phi h_{T}\right\|_{2}^{2}=\left|\left\langle\Phi h_{T}, \Phi h_{S}\right\rangle\right| \leqslant \sum_{j=1}^{M} x_{j}\left|\left\langle\Phi h_{T}, \Phi \mathbf{w}_{j}\right\rangle\right| \\
& \leqslant \sum_{j=1}^{M} x_{j} \theta_{k, k}\left\|h_{T}\right\|_{2}\left\|\mathbf{w}_{j}\right\|_{2} \leqslant \sum_{j=1}^{M} x_{j} \theta_{k, k}\left\|h_{T}\right\|_{2}\left\|h_{T}\right\|_{2} \\
& =\theta_{k, k}\left\|h_{T}\right\|_{2}^{2} .
\end{aligned}
$$

We have reached a contradiction. Hence $h=0$.

Remark 3.2 We state the proof for the $k$-sparse signal. In fact, one also can extend the proof to the noise case easily. In [1], Baker stated such a proof for the case where the signals are sparse in a redundant dictionary.

\section{References}

[1] Baker, C.A.: A note on sparsification by frames. arXiv:1305.6021 (2013). August

[2] Cai, T., Zhang, A.: Sharp RIP bound for sparse signal and low-rank matrix recovery. Appl. Comput. Harmon. Anal. 35, 74-93 (2013)

[3] Cai, T., Zhang, A.: Compressed sensing and affine rank minimization under restricted isometry. IEEE Trans. Signal Process. 61, 3279-3290 (2013)

[4] Cai, T., Zhang, A.: Sparse representation of a polytope and recovery of sparse signals and low-rank matrices. arXiv:1306.1154 (2013). June

[5] Cai, T., Wang, L., Xu, G.: Stable recovery of sparse signals and an oracle inequality. IEEE Trans. Inf. Theory 56, 3516-3522 (2010)

[6] Cai, T., Wang, L., Xu, G.: New bounds for restricted isometry constants. IEEE Trans. Inf. Theory 56, 4388-4394 (2010)

[7] Candès, E.J., Tao, T.: Decoding by linear programming. IEEE Trans. Inf. Theory 51, 4203-4215 (2005) 